\title{
Redução Endometrial por Vídeo-Histeroscopia: Experiência em um Hospital de Ensino
}

Endometrial Resection by Video-Hysteroscopy: Experience in a Teaching Hospital

Caio Parente Barbosa, Marcelo Ettruri Santos

Ana Cristina Napolitano, Paula Harue Tamanaka, Emerson Barchi Cordts

\section{RESUM0}

Objetivo: demonstrar a efetividade da redução endometrial vídeo-histeroscópica no tratamento do sangramento uterino anormal.

Métodos: foram analisados os prontuários de 60 pacientes com sangramento uterino anormal não-controlado clinicamente.

Resultados: oitenta e oito por cento das pacientes tiveram resposta adequada ao tratamento (53,3\% oligomenorréia e 35\% amenorréia). Foi encontrado um índice de 8,3\% de complicações (5 perfurações uterinas).

Conclusão: a redução endometrial vídeo-histeroscópica é uma técnica eficaz no tratamento do sangramento uterino anormal não-controlado clinicamente, com baixos índices de complicações intra e pós-operatórias.

PALAVRAS-CHAVE: Histeroscopia. Ressecção do endomiometrio. Endometrio hyperplana

\section{Introdução}

Nos Estados Unidos, cerca de 650.000 mulheres ao ano são submetidas a histerectomias, das quais 200.000 por indicação não-oncológica,

Serviço de Vídeo-histeroscopia Diagnóstica e Cirúrgica do Centro de Atenção Integral à Saúde da Mulher - Hospital de Ensino - Faculdade de Medicina do ABC, Santo André - SP. Correspondência:

Caio Parente Barbosa

Alameda Jauaperi, 732

Moema, CEP: 04523-013 São Paulo, SP sendo o sangramento uterino anormal e intratável a principal causa desse tipo de cirurgia ${ }^{1,3}$. No Reino Unido, de 50.000 histerectomias realizadas por ano, 18.500 são realizadas por causa de sangramento uterino anormal ${ }^{8}$.

A ablação endometrial vídeo-histeroscópica é um tratamento alternativo à histerectomia. Essg procedimento, minimamente invasivo, tem vantagens óbvias como menor morbidade e período de internação mais curto ${ }^{17}$. O endométrio pode ser retirado por meio de uma alça de ressecção, por coagulação com uma esfera no final do eletrodo (rollerball) ou por laser ${ }^{3,9,15}$. 
Em novembro de 1989, a "United States Food and Drug Administration" aprovou o uso de um ressectoscópio de fluxo contínuo em cirurgia ginecológica $^{16}$. Para essa ressecção foi utilizada corrente elétrica de alta freqüência de um gerador eletrocirúrgico que produzia corrente térmica na cavidade endometrial. Apesar disso, é atribuído a Goldrath, em 1981, o uso de Neodimiun-YAG laser sob controle histeroscópico para destruir o endométrio de pacientes com sangramento uterino anormal com o objetivo de diminuir ou mesmo abolir o sangramento anormal. Sucessivamente Decherney e Polan, em 1983, relataram a adoção de um ressectoscópio com eletrocoagulação unipolar de alta freqüência para alcançar o mesmo resultado ${ }^{10}$.

O uso do ressectoscópio com fluxo contínuo, utilizando corrente elétrica de alta freqüência com rollerball, foi primeiramente descrito por Vancaillie $^{17}$. Townsend et al. ${ }^{15}$, McLucas et al. ${ }^{9} \mathrm{e}$ Lefler et al. ${ }^{7}$ publicaram várias técnicas de ablação endometrial usadas no tratamento de sangramento anormal com útero anatomicamente normal. Os dois primeiros descreveram técnicas de ablação com coagulação com rollerball e Lefler descreveu técnica de eletrocoagulação com curetagem prévia e uso de vasopressina.

As terapias médicas têm demonstrado ser ineficazes em alguns casos. A terapia hormonal, com seus diversos efeitos colaterais, tornam-se mal suportadas com o tempo, e as curetagens uterinas, que por muitos anos foram utilizadas como método diagnóstico e/ou terapêutico, não mudam a sintomatologia a curto ou a longo prazo. A histerectomia parece ser ato desproporcionado, tendo em vista os riscos e morbidade operatória e custos em relação à ablação endometrial. Dessa maneira, a ablação endometrial tem tido a sua difusão favorecida entre os métodos terapêuticos ginecológicos.

Nosso estudo tenta determinar a efetividade da ablação vídeo-histeroscópica de endométrio como modalidade de tratamento dos sangramentos uterinos anormais, a partir dos resultados iniciais de 60 cirurgias realizadas na Faculdade de Medicina do ABC.

\section{Pacientes e Métodos}

Trata-se de um estudo retrospectivo, que compreende o período de agosto de 1993 a outubro de 1997, consistindo na análise dos prontuários de 60 pacientes com história de sangramento uterino anormal, resistente a tratamento clínico, que se submeteram à vídeo-histeroscopia operatória para ablação endometrial. As indicações para a cirurgia são mostradas na Tabela 1 .

Tabela 1 - Indicação de histeroscopia diagnóstica por sangramento uterino anormal.

\begin{tabular}{lcc}
\hline Sangramento uterino & n & $\%$ \\
\hline Hipermenorragia & 39 & 65,0 \\
Hipermenorréia & 06 & 10,0 \\
Menorragia & 11 & 18,3 \\
Metrorragia perimenopausa & 04 & 6,7 \\
\hline
\end{tabular}

A média de idade das pacientes do estudo foi de 42,3 anos (28 - 65), com 3,9 gestações em média $(1-18)$.

Todas as pacientes foram submetidas à histeroscopia diagnóstica com biópsia, excluindose qualquer patologia maligna. Em 13 pacientes foram diagnosticadas formações endocavitárias durante a histeroscopia diagnóstica: seis miomas submucosos e sete pólipos endometriais.

Todas as pacientes foram submetidas à histeroscopia operatória para ablação endometrial com ressectoscópio e coagulação com rollerball (descrita em Técnica Operatória). Em duas dessas pacientes foi realizada curetagem uterina prévia à histeroscopia, pois o padrão de proliferação endometrial foi julgado como fator de dificuldade para a realização do procedimento. Em 36 pacientes (60\%) foi realizado preparo prévio do endométrio com $150 \mathrm{mg}$ de acetato de medroxiprogesterona de depósito, intramuscular, por, pelo menos três meses que precederam a cirurgia.

Algumas pacientes foram submetidas a outras cirurgias durante o mesmo ato operatório: seis miomectomias histeroscópicas e sete polipectomias histeroscópicas.

Todas as pacientes foram submetidas à antibioticoprofilaxia com 4 doses de cefalotina $(1 \mathrm{~g}$ endovenoso, a cada 6 horas) iniciada na indução anestésica.

As pacientes tiveram controle histeroscópico ambulatorial pós-ablação de endométrio, após 3 (pelo menos) e 6 meses, investigando-se infecção endometrial, sinéquias em canal cervical (que poderiam promover o aparecimento de hematometra) e status menstrual.

\section{Técnica operatória}

Desde as primeiras tentativas até hoje foram utilizados diversos métodos para destruir completamente o endométrio.

Tomadas as devidas providências de assepsia e anti-sepsia, com a paciente em posição 
ginecológica, a cirurgia é iniciada com a dilatação do colo do útero com velas de Hegar até $9 \mathrm{~mm}$, introduzindo o ressectoscópio que é constituído de 2 camisas. A interna para infusão e a externa para aspiração do meio líquido de distensão intracavitária (solução de manitol a 3\%). Para distender a cavidade é utilizado um sistema de bomba específico que infunde o líquido a fluxo e pressão controlados que mantém a visão constantemente ideal da cavidade uterina. Com o ressectoscópio são possíveis a ressecção e a coagulação do endométrio ${ }^{1,3,10,12,13}$.

A ressecção consiste em retirar fatias de endométrio com cerca de 3 a $5 \mathrm{~mm}$ de espessura, sob visão endoscópica, por meio de um ressector em forma de $U$, conectado a uma fonte eletrocirúrgica.

Inicialmente é realizada cauterização do fundo e região cornual (periorificial) com rollerball e, depois, são ressecadas fatias de endométrio das paredes anterior, posterior e laterais direita e esquerda. Essa ressecção é feita desde o fundo uterino até o istmo, preservando-o, com 3 a $5 \mathrm{~mm}$ de profundidade, de modo a se retirar também uma fina camada de miométrio. As tiras de endométrio podem ser aspiradas ou retiradas através de curetas, devendo ser enviadas para exame anatomopatológico.

A ressecção é complementada com coagulação dos vasos sangrantes e cauterização dos focos restantes de endométrio por meio da utilização do rollerball.

Com essa técnica evita-se uma síndrome de Asherman, mantendo a cavidade uterina reepitelizada com endométrio da porção ístmica e sem aderências para que seja possível um seguimento adequado.

O preparo do endométrio para a cirurgia pode ser realizado com danazol 400 a $600 \mathrm{mg} /$ dia, via oral, por 4 a 6 semanas, análogos de GnRH (por exemplo, acetato de leuprolide de depósito - 3,75 mg, 2 doses, intramuscular a cada 30 dias) ou acetato de medroxiprogesterona de depósito na dose de $150 \mathrm{mg}$ intramuscular nos 2 ou 3 meses que precedem a cirurgia.

\section{Resultados}

Das 60 pacientes submetidas à ablação endometrial no Hospital de Ensino da Faculdade de Medicina do ABC, obtivemos os resultados apresentados a seguir:

Quatro pacientes tiveram indicação de ablação por hemorragia na perimenopausa $(6,7 \%)$, por hiperplasia simples de endométrio, sem atipias. Cinqüenta e seis pacientes $(93,7 \%)$ tiveram como indicação de ablação o sangramento uterino anormal que não melhorou com o tratamento clínico ou cirúrgico (curetagem uterina).

O período de internação variou de 1 a 3 dias, com média de 2,1 dias $( \pm 0,8)$. O tempo cirúrgico variou de 15 a 180 minutos, com média de 71,2 $\min ( \pm 42,7)$. A anestesia indicada foi a geral em 10 pacientes (18\%), 55,5\% raquianestesia e $26 \%$ anestesia peridural. Duas pacientes realizaram curetagem prévia à ablação endometrial e nas demais 58 pacientes esse procedimento não foi necessário.

As complicações ocorridas nesse grupo de pacientes compreenderam 5 perfurações uterinas, ou seja, $8,3 \%$.

A média de volume de manitol a 3\%, usado durante o procedimento foi de $7.700 \mathrm{ml}(2.000-$ $13.000 \mathrm{ml}$ ). A absorção de manitol ao final da cirurgia foi em média de $830 \mathrm{ml}(250-1.200 \mathrm{ml})$.

Todas as pacientes foram submetidas a profilaxia antibiótica com $1 \mathrm{~g}$ de cefalotina, iniciada na indução anestésica e mantida por 24 horas.

Os resultados dos exames anatomopatológicos mais encontrados foram: endométrio secretor $(35 \%)$, endométrio misto $(31,6 \%)$, hiperplasia simples do endométrio sem atipias $(16,6 \%)$, endométrio proliferado (15\%). Esses resultados são mostrados na Tabela 2 .

Tabela 2 - Resultados anatomopatológicos dos endométrios obtidos em 60 ablações histeroscópicas.

\begin{tabular}{lrr} 
Anátomopatológico & $\mathbf{n}$ & $\%$ \\
\hline Endométrio secretor & 21 & 35,0 \\
Endométrio proliferado & 9 & 15,0 \\
Endométrio misto & 19 & 31,6 \\
Hiperplasia glandular simples sem atipia & 10 & 16,6 \\
Hiperplasia glandular simples com atipia & 1 & 1,6 \\
Pólipo endometrial & 7 & 11,6 \\
Leiomioma & 5 & 8,3 \\
Adenomiose & 16 & 26,6 \\
\hline
\end{tabular}

Cinqüenta e oito pacientes $(96,7 \%)$ retornaram após três (pelo menos) e seis meses e apenas duas pacientes $(3,3 \%)$ não tiveram seguimento pós-operatório. No retorno as pacientes eram submetidas à histeroscopia de controle, ambulatorialmente, e também investigadas quanto aos ciclos menstruais, mostrados na Tabela 3. 
Tabela 3 - Evolução pós-operatória de 60 pacientes

\begin{tabular}{lrr}
\hline Evolução & n & $\boldsymbol{\%}$ \\
\hline Amenorréia & 21 & 35,0 \\
Diminuição do fluxo & 32 & 53,3 \\
Hipermenorragia & 5 & 8,3 \\
Perda de seguimento & 2 & 3,3 \\
\hline
\end{tabular}

\section{Discussão}

O sucesso de qualquer intervenção cirúrgica depende de inúmeros fatores, dentre eles a indicação cirúrgica adequada e um cirurgião capacitado e conhecedor da técnica a ser utilizada.

A ressecção endometrial está sendo utilizada em substituição às histerectomias nos casos de sangramento uterino anormal. Há situações em que grandes cirurgias são contra-indicadas, por envolver riscos durante a anestesia geral. A ressecção endometrial pode ser realizada sob anestesia espinhal e até mesmo sob bloqueio paracervical com sedação $0^{9,14}$. Neste estudo foram usadas anestesia geral $(18,5 \%)$ e anestesia espinhal $(81,5 \%)$. Essa última proporciona maior conforto e segurança pois é possivel diagnosticar, com maior facilidade, algumas complicações como as decorrentes do uso de manitol, quando absorvido maciçamente.

Considera-se o resultado como ideal para a técnica de ressecção endometrial com ressectoscópio quando as pacientes apresentam amenorréia, oligomenorréia ou tornam-se eumenorréicas, evitando intervenções cirúrgicas posteriores. Em nossa série de 60 pacientes, 32 $(53,3 \%)$ apresentaram diminuição do sangramento menstrual e 21 pacientes (35\%) amenorréia por um período de até seis meses. Dessa maneira, os $88,3 \%$ de resultados positivos são considerados adequados e iguais aos índices apresentados por outros autores que utilizaram a mesma técnica usada em nosso Serviço. Considerando-se um período de seguimento mínimo de 6 meses, a amenorréia ou hipomenorréia pode ocorrer de 50 a até mais de $90 \%$ dos casos. Tapper e Heinonen ${ }^{14}$ e Montagna e Zacchè ${ }^{11}$ observaram 25\% e $21 \%$ de amenorréia e $66 \%$ e $78 \%$ de diminuição do fluxo menstrual, respectivamente. Wortman e Daggett ${ }^{16}$ obtiveram índice de $97 \%$ (57\% de amenorréia, 40\% de oligomenorréia) e Alford e Hopkins s observaram amenorréia em $50 \%$ de suas pacientes.

Existem várias técnicas de ablação endometrial, e é evidente que as técnicas que ressecam alguns milimetros da espessura do miométrio promovem melhores resultados (maiores índices de amenorréia) daí decorrendo a variação de resultados na literatura.

Os resultados são tanto melhores quando se usam regimes hormonais para promover atrofia do endométrio, facilitando a realização da ablação endometrial histeroscópica ${ }^{1,12,16,17}$. Existe grande variação de esquemas hormonais que podem ser utilizados, no entanto, observam-se melhores resultados naquelas pacientes que utilizam danazol ou análogos do GnRH e resultados mais pobres quando é usada a progesterona de depósito, pela sua baixa absorção. Sessenta por cento de nossas pacientes utilizaram progesterona por 1 a 3 meses antes da cirurgia com a qual foi obtida atrofia endometrial, facilitando a realização da ressecção endometrial, além de promover menor sangramento durante a cirurgia. As demais nada utilizaram previamente à cirurgia e apenas em duas pacientes foi realizada curetagem uterina prévia, pois o nível de proliferação endometrial foi considerado impróprio para a realização da cirurgia.

Nosso tempo cirúrgico, que variou de 15 a 180 minutos, com média de $71,2 \mathrm{~min}( \pm 42,7)$ é alto quando comparado com os demais autores, cuja média variou de 20 a 45 minutos. Esses valores podem ser justificados pelo nosso Serviço estar inserido em um hospital de ensino de residentes, além de contarmos com outras cirurgias histeroscópicas (miomectomias e polipectomias) no mesmo ato anestésico. Nesse ponto, a observação de Alford $^{1}$ de que melhores resultados e também diminuição do tempo de intervenção cirúrgica dependem diretamente da experiência pessoal dos cirurgiões é bastante pertinente.

Alguns autores observaram que a presença de miomas submucosos, principalmente aqueles com mais de 50\% de componente intramural, operados no mesmo ato que a ressecção é um fator que favorece o insucesso da ablação endometrial ${ }^{6,15}$. Apenas 8,3\% de nossas pacientes apresentavam miomas com componente submucoso e nosso índice de resultados favoráveis $(88,3 \%)$ parece ainda estar dentro de limites esperados.

As complicações relacionadas à ablação endometrial são escassas. Podem compreender lesões viscerais e complicações infecciosas e sistêmicas. Na Tabela 4 podemos comparar algumas das principais complicações de diferentes autores.

A perfuração uterina, cujos índices chegam a $3 \%^{4,6,16}$, podem ser acompanhadas de outras lesões maiores de vísceras abdominais ${ }^{6}$. Nessa casuística 5 pacientes tiveram perfuração uterina $(8,3 \%)$. Apenas uma delas evoluiu para histerectomia imediata devido ao sangramento importante no local da perfuração; as demais pacientes não tiveram outras lesões maiores à 
videolaparoscopia realizada imediatamente após a perfuração.

Tabela 4 - Comparação das \% de complicações de ablações histeroscópicas de diversos autores.

\begin{tabular}{lrrcrr}
\hline Autores & $\mathrm{n}$ & $\begin{array}{r}\text { Perfuração } \\
\%\end{array}$ & $\begin{array}{c}\text { Infecção } \\
\%\end{array}$ & $\begin{array}{c}\text { Sangramento } \\
\%\end{array}$ & $\begin{array}{r}\% \text { total de } \\
\text { complicações }\end{array}$ \\
\hline Wortman $^{16}$ & 102 & 3,1 & 1,0 & 2,1 & 6,3 \\
Wortman $^{16}$ & 35 & - & - & - & - \\
Tapper $^{14}$ & 86 & 1,0 & - & - & 1,0 \\
Istre $^{6}$ & 412 & 2,0 & 7,3 & 6 & 15,3
\end{tabular}

A hemorragia intra ou pós-operatória é pouco comum, sendo uma intercorrência ligada mais à técnica, que destrói ou extrai o endométrio, e que pode ser tratada com ocitócicos, ou até mesmo tamponamento da cavidade uterina com o balão da sonda de Folley ${ }^{9}$. Não foi detectada hemorragia uterina importante em nenhuma das pacientes.

Neste estudo, nenhum caso de infecção intrauterina foi diagnosticado no pós-operatório. A incidência de infecção citada na literatura é bastante baixa, sempre inferior a $2 \%{ }^{2}$. O uso de antibióticoprofilaxia com cefalosporina por $24 \mathrm{~h}$, adotado neste estudo, pode ser responsável por tal resultado, apesar de ser procedimento ainda discutido pela maioria dos autores que alegam ser desnecessário pela baixa incidência das complicações infecciosas.

O consumo médio da solução de distensão da cavidade uterina é muito variável na literatura e pode ser mostrado e comparado na Tabela 5 . É de se esperar que com um tempo cirúrgico maior que de outros autores, haja, nos resultados aqui apresentados, maior consumo e conseqüentemente maior absorção da solução. Mesmo assim, não observamos em nossas pacientes, nenhuma intercorrência relacionada ao uso do manitol a 3\%. Muitos autores relatam como intercorrência a passagem para o compartimento intravascular do meio de distensão da cavidade uterina, embora essa seja uma intercorrência comum a todos os procedimentos cirúrgicos histeroscópicos. É necessário recordar que podem ocorrer situações muito graves como o edema agudo de pulmão e a encefalite irreversível, essa última nos casos de sobrecarga maciça da circulação porta. Nos casos menos graves podem ser observadas náuseas e hiponatremia que são corrigidas com correção do distúrbio hidroeletrolítico e medicação sintomática. Essas complicações parecem ser mais freqüentes quando são utilizadas técnicas de destruição ou de retirada de endométrio, favorecendo a exposição da vascularização endometrial e a passagem do meio de distensão ${ }^{11}$.
Tabela 5 - Consumo do líquido de distensão de acordo com diversos autores.

\begin{tabular}{|c|c|c|}
\hline \multirow[b]{2}{*}{ Autores } & \multicolumn{2}{|c|}{ Líquido de distensão $(\mathrm{ml})$} \\
\hline & infusão & Absorção \\
\hline Wortman ${ }^{16}$ & - & $760(0-2.000)$ \\
\hline Tapper $^{14}$ & $7.200(2.000-24.000)$ & $170(0-1.300)$ \\
\hline Istre $^{6}$ & 7.200 & 815 \\
\hline
\end{tabular}

O tempo de internação das pacientes foi de 2 dias, em média $( \pm 0,8)$, diferindo do tempo referido pelos autores, cujo período médio de internação foi de 24 horas, sendo muitas cirurgias realizadas em regime de hospital dia $^{5,17}$.

Ao exame histopatológico do material das 60 ressecções endometriais foi encontrada maior porcentagem de endométrio secretor (35\%) e apenas $15 \%$ de endométrio proliferativo. Os autores consultados (Tabela 6) mostram, ao contrário, maior freqüência do diagnóstico de endométrio proliferativo ${ }^{15,16,17}$. Pode-se dizer que esses resultados diferem dos nossos, pois há maior tendência dos autores em operar as pacientes logo após a menstruação, portanto, na fase proliferativa inicial. Já com as pacientes deste serviço isso nem sempre é possivel, pois dependemos de leitos públicos e de lista de espera na programação cirúrgica.

Tabela 6 - Resultados anátomo-patológicos: comparação de resultados.

\begin{tabular}{|c|c|c|c|c|c|c|}
\hline \multirow{2}{*}{$\begin{array}{l}\text { Anatomo- } \\
\text { patológico }\end{array}$} & \multicolumn{2}{|c|}{ FМАВС(1997) } & \multicolumn{2}{|c|}{ Wortman $^{16}$} & \multicolumn{2}{|c|}{ Tapper $^{14}$} \\
\hline & $\mathbf{n}$ & $\%$ & $\mathbf{n}$ & $\%$ & $\mathbf{n}$ & $\%$ \\
\hline Endométrio secretor & 21 & 35,0 & 16 & 46,0 & 16 & 19 \\
\hline Endométrio proliferado & 9 & 15,0 & 14 & 40,0 & 45 & 53 \\
\hline Endométrio misto & 19 & 31,6 & 3 & 8,6 & 5 & 6 \\
\hline Hiperplasia simples sem atipia & a 10 & 16,6 & 2 & 5,7 & 1 & 1 \\
\hline Hiperplasia simples com atipia & ia 1 & 1,6 & - & - & - & \\
\hline Pólipo endometrial & 7 & 11,6 & - & - & 3 & 4 \\
\hline Leiomioma & 5 & 8,3 & - & - & 19 & 22 \\
\hline Adenomiose & 16 & 26,6 & 7 & 20,0 & 8 & 9 \\
\hline
\end{tabular}

Pelo exposto, a redução endometrial histeroscópica é um método eficaz para tratamento de sangramento uterino anormal, não controlado clinicamente, com vantagens evidentes: menor tempo de hospitalização, menor morbidade e baixo índice de complicação.

\section{SUMMARY}

Objective: to demonstrate the effectiveness of videohysteroscopic endometrial resection in the treatment of 
abnormal uterine bleeding.

Patients and method: The authors studied 60 records of patients with abnormal uterine bleeding who did not respond to clinical treatment.

Results: eighty-eight percent of the patients had adequate response to the treatment (53\% oligomenorrhea and $35 \%$ amenorrhea). The complication rate was $8.3 \%$ (5 uterine perforations).

Conclusion: video-hysteroscopic endometrial resection is an effective technique to treat abnormal uterine bleeding which failed to respond to clinical management. The intra and postoperative complication rates are low.

KEY WORDS: Hysteroscopy. Endometrial hyperplanaresection.

\section{Referências}

1.Alford WS, Hopkins MP. Endometrial rollerball ablation. J Reprod Med 1996; 41: 251-4.

2.Amin-Hanjani S, Good JM. Pyometra after endometrial resection and ablation. Obstet Gynecol 1995; 85: 893-4.

3.Brooks PG. Hysteroscopic surgery using the resectoscope: myomas, ablation, septae e synechiae. Does preoperative medication help? Clin Obstet Gynecol 1992; 45: 249-55.

4.Cravello L, Montgolfier R, D’Ercole C, Boubli L, Blanc B. Hysteroscopic surgery in postmenopausal women. Acta Obstet Gynecol Scand 1996; 75: 563-6.

5.Goldenberg M, Sivan E, Bider D, Mashiach S, Seidman DS. Endometrial resection vs. abdominal hysterectomy for menorrhagia. Correlated sample analysis. J Reprod Med 1996, 41: 333-6.

6.Istre O. Transcervical resection of endometrium and fibroids: the outcome of 412 operations performed over 5 years. Acta Obstet Gynecol Scand 1996; 75: 567-74.
7.Lefler HT, Sullivan GH, Hulka JF. Modified endometrial ablation: electrocoagulation with vasopressin and suction curetage preparation. Obstet Gynecol 1991; 77: 949-53.

8.Magos AL, Baumann R, Lockwood, GM, Turnbull, A C. Experience with the first 250 endometrial resections for menorrhagia. Lancet 1991; 337: 1074-79.

9.Mclucas B. Endometrial ablation with the rollerball electrode. J Reprod Med 1990; 35: 1055-61.

10.Moir CL, Mandin H, Brant R.Sorbitol 2,5\% manitol $0,54 \%$ irrigation solution for hysteroscopic endometrial ablation surgery. Can J Anaesth 1997; 44: 473-78.

11.Montagna S, Zacchè G. L'ablazione endometriale revisione delle tecniche e risulti. Minerva Ginecol 1993; 45: 409-17.

12.Montagna S, Zacchè G. L’ablazione endometriale com resettoscopio - esperienza personale. Minerva Ginecol 1995; 47: 17-21.

13.Pyper RGD, Haery AD. A review of 80 endometrial resections for menorrhagia. Br J Obstet Gynaecol 1991; 98: 1049-54.

14.Tapper AM, Heinonen PK. Hysteroscopic endomiometrial resection for the treatment of menorrhagia - follow-up of 86 cases. Obstet Gynecol 1995. 62: 75-9.

15.Townsend DE, Richart, RM, Paskowitz R. A rollerball coagulation of the endometrium. Obstet Gynecol 1990; 76: 310-3.

16.Wortman M, Dagget A. Hysteroscopic endomiometrial resection: A new technique for the treatment of menorrhagia. Obstet Gynecol, 1994; 83: 295-8.

17.Vancaillie TG. Electrocoagulation of the endometrium with the ball-end resectoscope (rollerball). Obstet Gynecol, 1989; 74: 425-9. 